\title{
Neurogenetic Disease: Genes, Mechanisms, and Future Promise
}

\author{
William T. Dauer
}

Published online: 27 September 2014

(C) The American Society for Experimental NeuroTherapeutics, Inc. 2014

The last decade has witnessed an explosion of genetic discovery, transforming essentially all aspects of how we categorize disease and conceptualize its pathogenesis. These discoveries are increasingly affecting clinical care in myriad ways, from diagnosis to prognosis to choice of therapy. The era of personalized medicine has not yet arrived, but individual triumphs in this direction mount, giving hope that the ever accelerating wave of genetic discovery - and the insights these discoveries enable - are moving us in that direction.

Like most other areas of medicine, our understanding and treatment of neurological disease is being transformed by recent genetic discoveries. Whereas only approximately 15 years ago prevalent scourges such as Alzheimer (AD) or Parkinson diseases (PD) were believed to be largely or completely "idiopathic" in origin, the considerable impact of genetic risk in the development and progression of these illnesses is increasingly appreciated, with some estimates placing the genetic risk of PD as high as $\sim 50 \%$ [1]. These discoveries are similarly affecting pediatric neurology, enabling many more diagnoses to be made in cases previously resistant to molecular classification, and focusing researchers of these illnesses in new directions.

The goal of this special issue on neurogenetics, "Neurogenetic Disease: Genes, Mechanisms, and Future Promise," is to highlight key developments, focusing on 4 areas: tools and general mechanisms; adult neurodegenerative disease; pediatric neurogenetic disease; and gene therapy.

The section on tools and general mechanisms begins with a review by Lohmann and Klein [2] of next generation sequencing ("NextGen"), arguably the most important recent development in this area. NextGen sequencing is enabling large-scale whole exome and genome screening of patient

W. Dauer $(\bowtie)$

Cell and Developmental Biology, University of Michigan Medical

School, 109 Zina Pitcher Place, BSRB, Room 4003, Ann Arbor, MI 48109-2200, USA

e-mail: dauer@med.umich.edu populations of a sort unimaginable in the recent past, and is a key methodology for the hoped for future of truly personalized medicine. NextGen sequencing has dramatically reduced the cost of gene sequencing, rapidly bringing us to the era of the "\$100 genome," which will eliminate the one-by-one search for individual genetic mutations clinicians have traditionally employed in diagnosis. This technology is also responsible, in part, for the discovery of new genetic mechanisms controlling biological processes, which appear to be important contributors to neurological disease when disrupted. Epigenetics - strictly the study of chemical changes to DNA that alter its structure and function (e.g., methylation and related modifications) - has benefited tremendously from NextGen sequencing, enabling several novel modulators of transcription and translation to be discovered. As reviewed by Qureshi and Mehler [3], several defects in epigenetic control mechanisms have been linked to disease pathogenesis, and the ability to use NextGen is allowing investigation of epigenetic mechanisms on a population level ("epigenetic epidemiology"). Another recent discovery of genetic control is repeatassociated non-AUG ("RAN") translation, which has been implicated in several neurodegenerative diseases-most prominently in amyotrophic lateral sclerosis where it may account for $5-10 \%$ of nonfamilial (previously considered idiopathic) cases. Kearse and Todd [4] review the current state of understanding of RAN translation, including its involvement in normal cellular function and role in the pathogenesis of neurodegeneration.

The section on adult neurodegenerative disease focuses on how genetic discoveries have advanced understanding of the pathogenesis of individual neurodegenerative diseases. Guerreiro and Hardy [5] review current knowledge of the genetic architecture of AD. Extending the technology theme of the previous section, they also discuss the transition from traditional "family-based" Mendelian studies to secondgeneration studies that increasingly rely on NextGen and 
related sequencing methods, and consider the role of such studies for future investigations of AD genetics. As noted above, genetic discoveries have altered our conception of PD perhaps more than any other illness. Dominant mutations in LRRK2 encoding leucine-rich repeat kinase 2 are the most common inherited form of PD, accounting for up to $5 \%$ of nonfamilial cases and in defined populations (e.g., Ashkenazi Jews) are responsible for up to one-third of cases. LRRK2 is a large multifunctional protein, which has resisted facile analysis. Rudenko and Cookson [6] review a range of studies on this complex protein, and propose a unifying model for how disparate LRRK2 mutations may affect a common downstream pathway. Studies of polyglutamine (polyQ) disease have been instrumental in revealing mechanisms of neurodegeneration that are broadly relevant. One such mechanism that is gaining in interest and importance is the role of early electrophysiological changes that precede neurodegeneration. These changes appear to contribute to disease symptoms, and being "upstream" of cell death, present an excellent target for novel therapeutics. Chopra and Shakkottai [7] review a range of work in this burgeoning field, emphasizing shared mechanisms across polyQ diseases, and future directions for mechanistic understanding and potential therapeutic development.

Pediatric disease, the focus of the third section, presents an ideal scenario for advances in neurogenetics: the patient's close relatives are typically available for study, and the common desire for additional children strengthens the importance of genetic diagnosis considerably. One outcome of genetic discoveries in this area has been to highlight organelle dysfunction as a useful way to categorize and conceptualize disease. The first two contributions to this section reveal how genetic defects that disrupt nuclear membrane or muscle triad function can cause neuromuscular disease, including muscular dystrophy $[8,9]$. Disruption of the nuclear membrane has also been implicated in the pathogenesis of DYT1 dystonia, which is reviewed together with other genetic forms of isolated inherited dystonia [10]. Also reviewed in this section are two diseases notable for being tremendously debilitating or frequently fatal: spinal muscular atrophy (SMA) and epileptic encephalopathy (EE). In their paper on SMA, Awano et al. [11] review how gene discovery can lead to the development of faithful animal models of disease, and how results from these models and human genetic studies can focus therapeutic efforts, which are currently underway for this dreaded illness. Esmaeeli Nieh and Sherr [12] outline the various forms of EE and their treatments, and review how NextGen sequencing of large numbers of these children together with their parents is revolutionizing our understanding of the genetic topography of this group of disorders. Importantly, these new studies highlight how mutations in genes other than the typical ion channel culprits cause many of these syndromes - information sure to greatly influence future study of these diseases.

The overwhelming contribution of neurogenetics thus far has been on the diagnosis and classification of neurological disease and, through the study of "disease genes," a revolution in our understanding of disease pathogenesis. The great hope for the future is that this information can be translated into similarly revolutionary therapies, though the pace of therapeutic development has been disappointing. Nevertheless, new delivery vehicles and methods for precise gene editing have fueled progress and justifiable excitement in this area, as reviewed by Maguire et al. [13] in the section on gene therapy. The hope is that the tremendous advances in genetic understanding and technology will finally "close the loop," and that a future consideration of neurogenetics in this journal will be dominated by reviews of meaningful developments in therapy.

I thank the authors who have invested tremendous energy and their valuable time and wisdom to this effort. Equally important to the success of this project have been Editorial Manager Linda Powell and Editor-in-Chief Maral Mouradian—thank you!

Required Author Forms Disclosure forms provided by the authors are available with the online version of this article.

\section{References}

1. Hardy J. Genetic analysis of pathways to Parkinson disease. Neuron 2010;68:201-206.

2. Lohmann K, Klein C. Next generation sequencing and the future of genetic diagnosis. Neurotherapeutics 2014;1-9. doi:10.1007/s13311014-0288-8.

3. Qureshi IA, Mehler MF. Epigenetic mechanisms underlying the pathogenesis of neurogenentic diseases. Neurotherapeutics 2014. doi:10.1007/s13311-014-0302-1.

4. Kearse MG, Todd PK. Repeat associated non-AUG translation and its impact in neurodegenerative disease. Neurotherapeutics 2014;199. doi:10.1007/s13311-014-0292-Z.

5. Guerreiro R, Hardy J. Genetics of Alzheimer's Disease. Neurotherapeutics 2014. doi:10.1007/s13311-014-0295-9.

6. Rudenko IN, Cookson MR. Heterogeneity of leucine-rich repeat 2 mutations: Genetics, mechanisms, and therapeutic implications. Neurotherapeutics 2014;1-13. doi:10.1007/s13311-014-0284-z.

7. Chopra R, Shakkottai VG. The role for alterations in neuronal activity in the pathogenesis of polyglutamine repeat disorders. Neurotherapeutics 2014. doi:10.1007/s13311-014-0289-7.

8. Worman HJ, Dauer WT. The Nuclear Envelope: An Intriguing Focal Point for Neurogenetic Disease. Neurotherapeutics 2014. doi:10. 1007/s13311-014-0296-8.

9. Dowling JJ, Lawlor MW, Dirksen RT. Triadopathies: An emerging class of skeletal muscle diseases. Neurotherapeutics 2014;1-13. doi: 10.1007/s13311-014-0300-3.

10. Dauer W. Inherited Isolated Dystonia: Clinical Genetics and Gene Function. Neurotherapeutics 2014. doi:10.1007/s13311-014-0297-7.

11. Awano T, Kim J-K, Monani UR. Spinal muscular atrophy: Journeying from bench to bedside. Neurotherapeutics 2014;1-10. doi:10.1007/s13311-014-0293-y.

12. Esmaeeli Nieh S, Sherr EH. Epileptic encephalopathies: New genes and new pathways. Neurotherapeutics 2014. doi:10.1007/s13311014-0301-2.

13. Maguire CA, Ramirez SH, Merkel SF, Sena-Esteves M, Breakefield XO. Gene Therapy for the Nervous System: Challenges and New Strategies Neurotherapeutics 2014. doi:10.1007/s13311-014-0299-5. 